\title{
THE JAWS AND TEETH IN PAGET'S DISEASE OF BONE
}

\author{
BY \\ R. B. LUCAS \\ From the Department of Pathology, Royal Dental Hospital of the London School \\ of Dental Surgery
}

(RECEIVED FOR PUBLICATION JANUARY 18, 1955)

\begin{abstract}
That Paget's disease of bone may affect the jaws has been known since Moore's (1923) report of a case in which there was a bony tumour of the maxilla together with osteoporosis circumscripta of the frontal region, though until quite recently the disease in this site has been considered rare. Osteoporosis circumscripta itself has now been identified with an early phase of Paget's disease (Sosman, 1927 ; and others), and a number of cases have since been described in which there has been a coexisting lesion in the maxilla (Kasabach and Gutman, 1937 ; Elkeles, 1947 ; Rushton, 1948). It
\end{abstract} has also been realized that the symptoms arising from the lesions in the jaws may be the first of which the patient complains, leading to detection of lesions in other parts of the skull or in other bones (Novak and Burket, 1944 ; Jacobs, 1945), or that such lesions may be the only ones present (Thoma, Howe, and Wenig, 1945).

The incidence of jaw lesions is uncertain, as most of the published reports concern single cases or relatively small series, with the exception of the report by Stafne and Austin (1938), who found 23 cases with mandibular or maxillary involvement out of 138 cases in which the skull was affected. The maxilla is much more often involved than the mandible ; in the series just referred to the mandible was involved in only three of the 23 cases. Instances of mandibular involvement have also been reported by Seldin (1933), Glickman (1943), Jacobs (1945), Thoma et al. (1945), and Stafne (1946).

The teeth are often affected in association with the surrounding bone, the principal abnormality being hypercementosis (Fox, 1933). As a result of enlargement of the jaws and of the overgrowth of cementum which may lead to ankylosis of the involved teeth and difficulty in their extraction, it not infrequently happens that the patient with Paget's disease is first seen by the dental surgeon. Thus the specimens which the pathologist receives for histological examination may be teeth or fragments of bone from the jaws, and it is the purpose of this paper to describe the appearances in these tissues when affected by Paget's disease. The materials from which these observations have been made comprise teeth and maxillary bone from six patients who complained of dental symptoms in the first instance.

\section{Clinical Features}

The patient with Paget's disease affecting the jaw complains of progressive enlargement of the maxilla or, much less frequently, the mandible. As a result of the increase in size in the dental arch the teeth become spaced, and if dentures are worn these cease to fit properly. The disproportion in size between the maxilla and the mandible gives rise to the inverted triangle type of facial contour.

The overgrowth of cementum on the roots of the teeth may lead to difficulty in extraction; the patient may be first referred to hospital on this account. Or following extractions persistent sinuses may develop, as the dense, avascular areas of bone which form in the osteosclerotic phase of the disease appear to be particularly prone to infection (Burket, 1946).

Pain occasionally occurs in connexion with the jaw lesions, sometimes of considerable severity.

\section{Histological Observations}

Bone.-Microscopic examination of bone from the jaws shows the characteristic changes of Paget's disease as seen in other parts of the skeleton. These are due essentially to a combination of destruction and repair, occurring without relation to the statics or dynam:cs of the involved bone (Weinmann and Sicher, 1947).

Teeth.-That the teeth may be involved in Paget's disease has been recognized since Fox's 


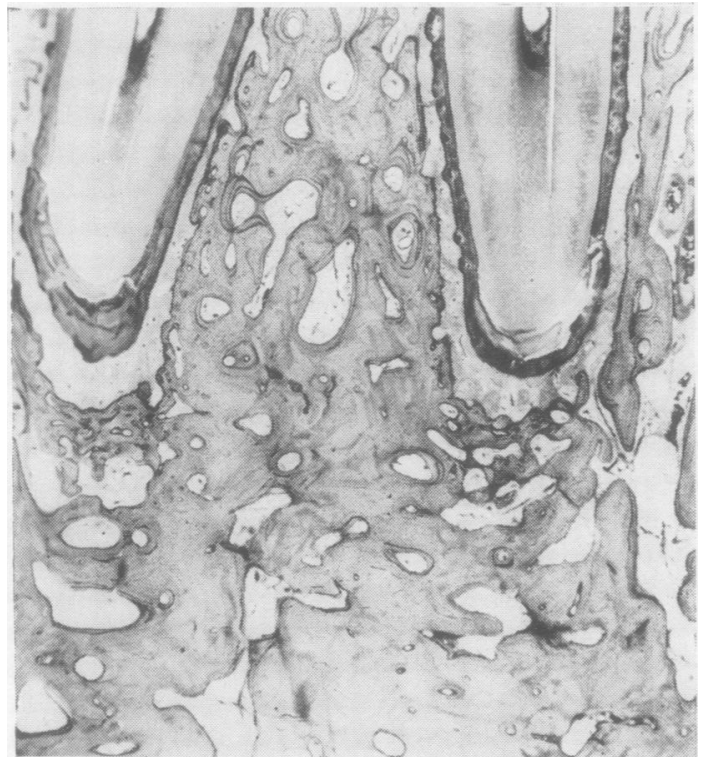

FIG. 1.-The apices of two normal teeth in situ in the alveolus, $\times 9$

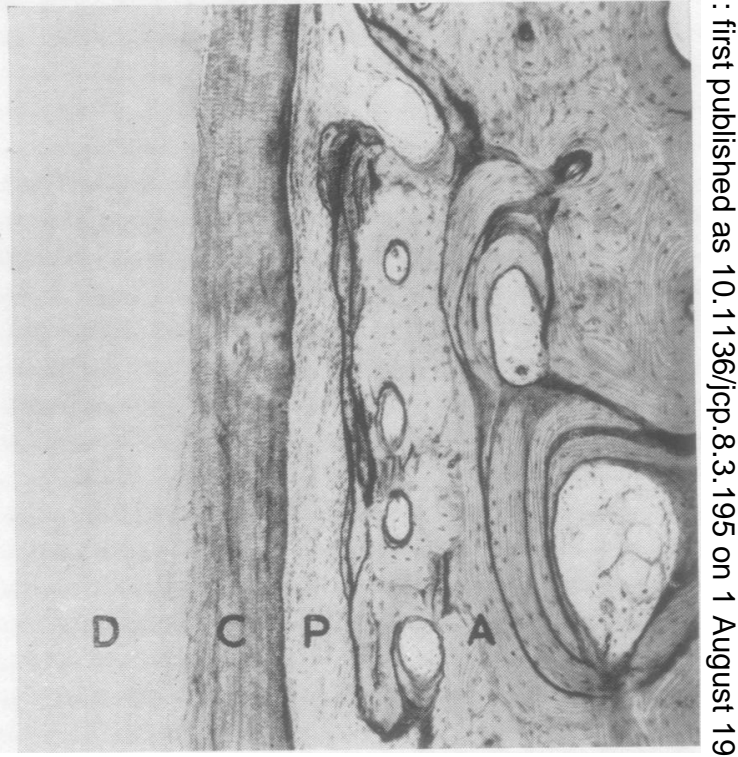

FIG. 2.-Higher magnification of a feld from Fig. 1. D, dentine $\mathrm{C}$, cementum; P, periodontal membrane; A, alveolar bone, $\times 50$.

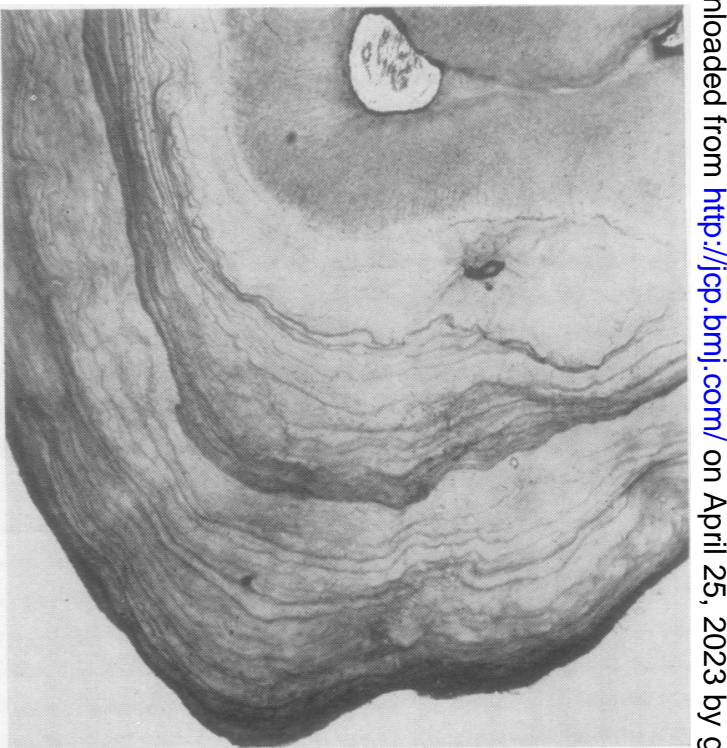

FIG. 4.-Apex of tooth shown in Fig. 3, to demonstrate the lamella arrangement of the cementum, $\times 50$.

other authors have since reported similar findings Descriptions of the histopathology of the teetlo are scanty. Rushton (1938) reports the histologica霖 findings in three teeth from two cases of Paget' $\mathbb{R}$ disease affecting the jaws, and Thoma (1950) als describes the teeth in two cases.
(1933) description of a case in which the maxilla was affected, and the roots of all the maxillary teeth showed, radiographically, the presence of knob-like irregularities. Stafne and Austin (1938) showed that hypercementosis of the teeth is a characteristic finding in Paget's disease, and many 
The Normal Tooth.-Fig. 1 shows the apical portions of two teeth, in situ in the alveolus, and demonstrates the nature of their attachment to the jaw. Fig. 2 is a higher magnification to show detail. The dentine, $\mathrm{D}$, is lined by a thin shell of cementum, $\mathrm{C}$, in which are embedded the connective tissue fibres of the periodontal membrane, $\mathbf{P}$. This membrane acts as a suspensory ligament for the tooth, attaching it to the alveolus, $\mathbf{A}$.

Cementum is a modified type of bone covering the roots of the teeth, two forms being recognized, acellular and cellular. Acellular cementum consists of calcified tissue in which is embedded the Sharpey's fibres of the periodontal membrane ; it contains no cell spaces or cells. Cellular cementum contains cementocytes lying in lacunae, and is therefore similar to bone. Acellular cementum is normally laid down on the surface of dentine, as in Figs. 1 and 2, while cellular cementum is usually formed on the surface of acellular cementum, but the layers of the two types may alternate in almost any arrangement (Orban, 1949). Both types of cementum are separated by incremental lines.

Hypercementosis.-The term hypercementosis is used to denote an abnormal thickening of the cementum, which may be diffuse or may be confined to one part of the tooth only. Paget's disease is a relatively uncommon cause of the condition, which occurs frequently as a result of other factors, such as infection, but the presence of hypercementosis in a patient in the appropriate age group should always lead to consideration of the possibility of Paget's disease.

Fig. 3 shows an example of hypercementosis due to periapical infection. The apical two-thirds of the tooth is ensheathed in a relatively thick mass of cementum, laid down in lamellae. The incremental lines are clearly seen in the higher magnification of Fig. 4, and it will be noticed that these form a series of more or less regular layers. Nowhere is there any suggestion of mosaic pattern.

Another example of hypercementosis due to infection is shown in Figs. 5 and 6 . Though the cementum has been deposited more regularly in this case than in the preceding specimen there is no evidence of the typical curvilinear markings of Paget's disease.

Paget's Disease.-The upper premolar tooth shown in Fig. 11 was obtained from a man aged 63 years, who complained of pain in the upper jaw following dental extractions seven weeks previously. He also complained of increasing deafness and tinnitus. On examination there was marked enlargement of the maxillary tuberosities and pronounced prominence of the labial maxilla.
Radiological examination showed diffuse woolly sclerosis over much of the maxilla and the calvarium was thickened. The serum alkaline phosphatase was 59 units.

Histological examination of the tooth shows hypercementosis (Fig. 7). The increase in cementum involves both roots of the tooth and is diffusely distributed except at one point, where the hyperplastic tissue forms a spur-like projection. Immediately above this projection there remain some fibres of the periodontal membrane, containing two small fragments of alveolar bone. Higher power magnification (Fig. 8) shows the spur-like structure to consist of laminae of cellular and acellular cementum. These are laid down for the most part in quite regular layers, but at about the centre of the photograph there can be seen an area where the cement lines are irregular and pronounced, suggesting a mosaic appearance. One of the fragments of alveolar bone, seen in higher magnification in Fig. 9, likewise shows the curvilinear markings associated with Paget's disease.

These findings are of course minimal, and would of themselves hardly constitute sufficient evidence for a diagnosis of Paget's disease. Nevertheless, they are sufficient to arouse suspicion, so that full radiological and appropriate biochemical examinations are undertaken. A further biopsy was performed in this case, a portion of alveolar bone being removed. This showed the typical changes of Paget's disease.

The maxillary molar teeth shown in Figs. 10 and 11 were removed from a woman aged 73 years, who complained of pain in both upper canine regions, and of increasing deafness of some years' duration. Radiological examination showed gross hypercementosis of the maxillary molar teeth and the typical appearances of Paget's disease in the bones of the cranium. The serum alkaline phosphatase was 27 units.

Histological examination of the teeth reveals a striking appearance due to the masses of cementum which have been laid down around the roots. This tissue has been deposited in an irregular manner, quite unlike the lamellation which normally takes place. Figs. 12 and 13 are higher magnifications of the root area and cementum of the specimen seen in Fig. 11 and show the broad, deeply staining cement lines indicating the complicated cycle of deposition and resorption of cementum which has taken place. This activity has produced a picture quite different from that seen in the examples of cementum overgrowth due to infection shown in Figs. 4 and 6. In the former, the excessive cementum has been laid down in lamellae, while in the latter, though the lamellar 


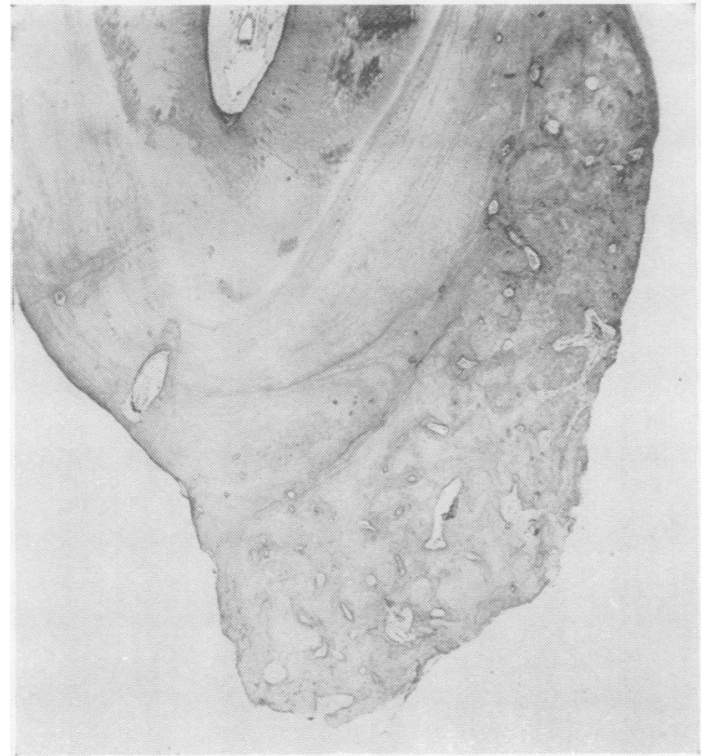

Fig. 5

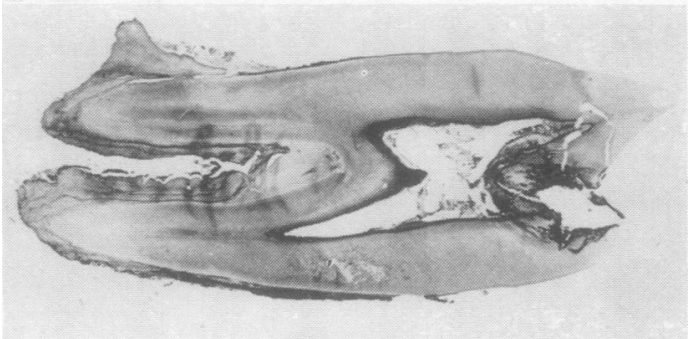

Fic. 7

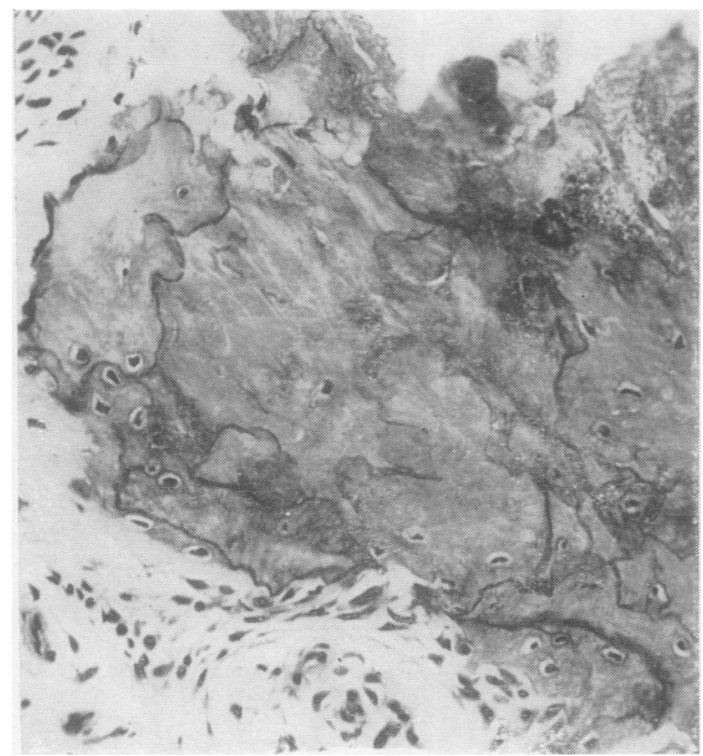

Fig. 9
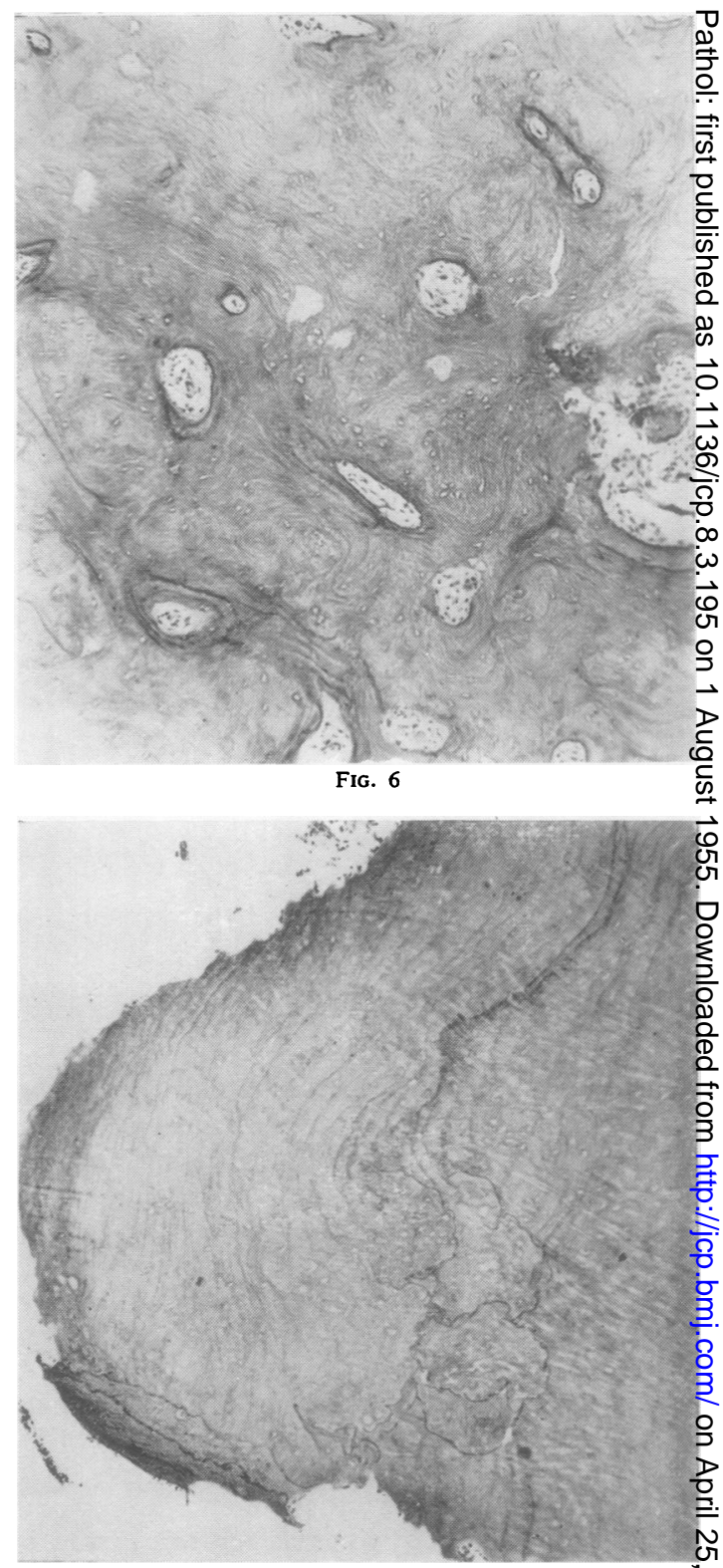

Fig. 8

กิ

FIG. 5.-Apex of tooth showing hyperzenentosis due to infection, $\times 9$.

FIG. 6. - Higher magnification of a field from Fig. $5, \times 50$ 을

FIG. 7.-Tooth from a case of Paget's disease showing $\overline{\mathbb{D}}$ hypercementosis of both roots, with a spur-like projection in one area, $\times 3.5$.

To FIG. 8.- Higher magnification of the spur shown in Fig. 7 鬲
Irregular cement lines are seen, $\times 50$.

FIG. 9.-Fragment of bone from the periodontal mem brane of the tooth shown in Fig. 7. The cement lines show a mosaic appearance, $\times 200$. 


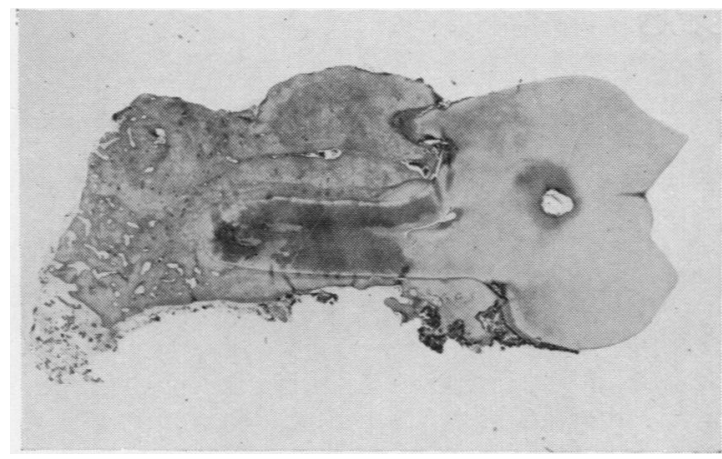

Fig. 10

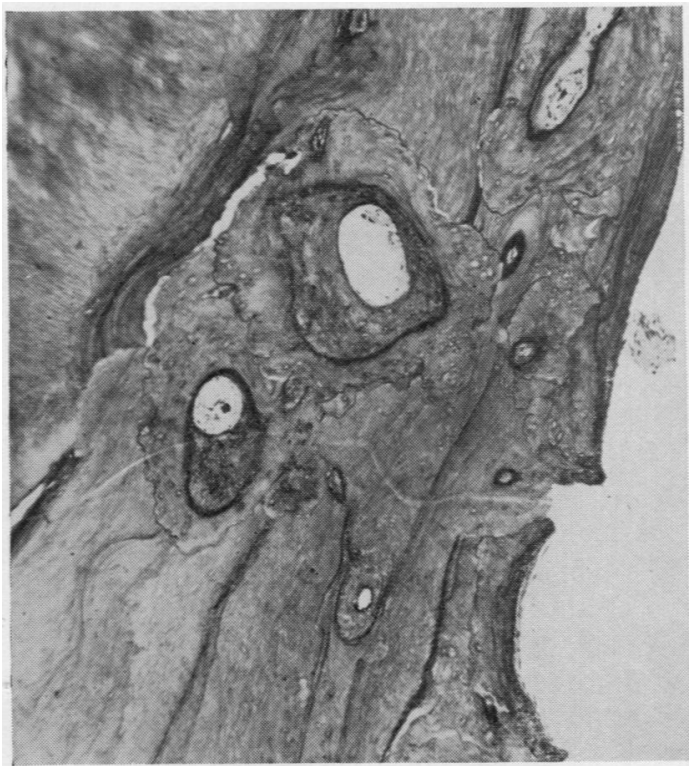

Fig. 12

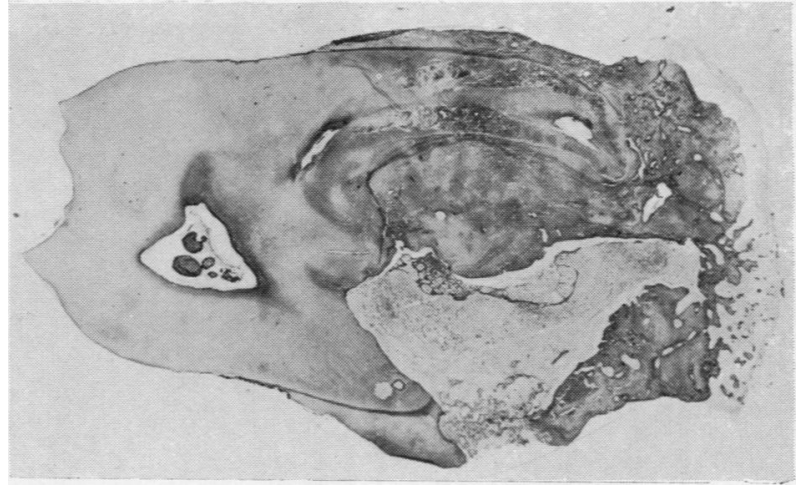

Fig. 11

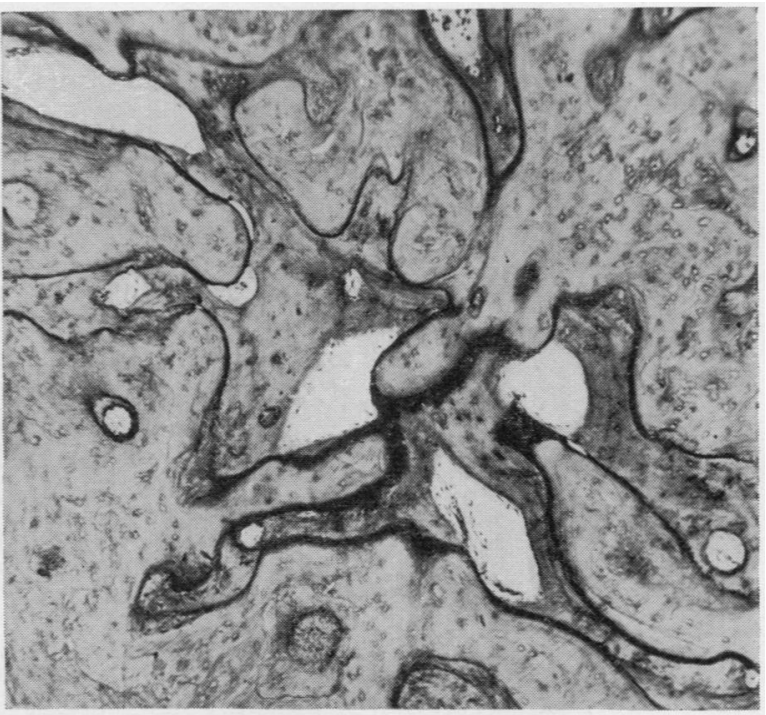

Fio. 13

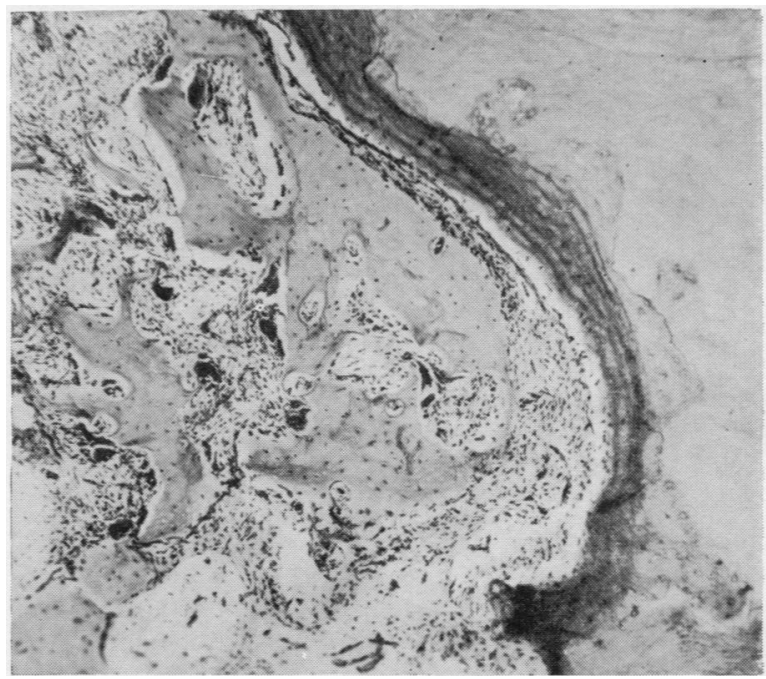

FIg 14 
arrangement is lacking, the thick mass of cementum does not show curvilinear markings.

Another feature in the pathological process is seen in Fig. 14. This is a field from Fig. 11, showing resorption of dentine with repair by comparatively normal cementum and ingrowth of active Paget bone.

\section{Discussion}

When Paget's disease involves the jaws, it may do so as part of a more general affection of the skull and other parts of the skeleton. In cases of this type the diagnosis is generally made on clinical grounds, together with the results of radiological and biochemical examinations. The pathologist may receive portions of bone for confirmatory examination, or sometimes bone is removed from the enlarged maxilla or mandible for prosthetic reasons.

In other cases the jaws alone may be affected, and the clinical and radiographic appearances may be interpreted as those of chronic osteomyelitis. In such cases histological examination of bone or teeth leads to the correct diagnosis or where the appearances are suggestive, if not conclusive, to the performance of biochemical tests and adequate follow-up of the patient. Too great reliance, however, should not be placed upon the biochemical tests ; Cahn (1948) points out that these may be normal if the disease is localized to one small area, for example, the maxilla.

In the five cases in the present series in which bone was examined, there was little difficulty in arriving at a histological diagnosis. Jaffe (1933) has pointed out, however, that the bone of the jaws contains more cement lines than does bone from other parts, and that these are often irregularly disposed, presumably because the stresses of mastication occasion a very active turnover of bone. He also mentions healing infectious periostitis with excessive bone formation as a possible cause of numerous cement lines and generalized osteitis fibrosa cystica as a cause of chaotic bony architecture. But when mosaics appear in condi $\stackrel{-}{\Rightarrow}$ tions other than Paget's disease their extent isos limited and the cement lines tend to be moreo regular.

Where teeth only are available for examination, $\frac{\bar{\sigma}}{\sqrt{5}}$ a histological diagnosis may still be made with con- $\frac{\mathbb{Q}}{\Omega}$ fidence when the cementum shows the changes justo described. Curvilinear markings of that type are ${ }^{\infty}$ not found in cementum proliferation in any con- $\overrightarrow{0}$ dition other than Paget's disease. Rushton's (1938) cases showed similar cemental changes and Thoma's (1950) cases probably do too, so far as요 can be judged from his illustrations. The pointo to which Thoma draws attention, however, is theit extensive resorption of both cementum and dentine in his specimens, and the growth into the excavatedor area of trabeculae of typical Paget bone.

\section{Summary}

Paget's disease of bone may affest the jaws and teeth, sometimes as part of a more general affec- $-\overrightarrow{0}$ tion of the skull or skeleton, sometimes as a soli-g tary lesion. The microscopic appearances of the teeth and alveolar bone are described and the diagnosis discussed.

\section{REFERENCES}

Burket, L. W. (1946). Oral Medicine. Lippincott, Philadelphia. Cahn, L. R. (1948). Oral Surg., 1, 917.

Elkeles, A. (1947). Proc. roy. Soc. Med., 40, 466.

Fox, L. (1933). J. Amer, dent. Ass., 20, 1823.

Glickman. I. (1943). Amer. J. Orthodont. (Oral Surg. Sect.), 29, 591

Jacobs, M. H. (1945). Ibid.. 31, 104.

Jaffe, H. L. (1933). Arch. Path., Chicago, 15, 83.

Kasabach, H. H., and Gutman, A. B. (1937). Amer. J. Roentgenol. 37, 577.

Moore, S. (1923). Ibid., 10, 507.

Novak, A. J., and Burket, L. W. (1944). Amer. J. Orthodont. (Ora Surg. Sect.), 30, 544 .

Orban, B. (1949). Oral Histology and Embryology, 2nd ed. Mosby St. Louis.

Rushton, M. A. (1938). Guy's Hosp. Rep., 88, 163.

(1948). Brit. dent. J., 84, 189.

Seldin, N. A. (1933). Dent. Cosm., 75, 691

Sosman, M. C. (1927). Radiology, 9, 396.

Stafne, E. C. (1946). J. oral Surg., 4, 114.

and Austin. L. T. (1938). J. Amer. dent. Ass., 25, 1202.

Thoma, K. H. (1950), Oral Pathology, 3rd ed. Mosby, St. Louiso

Howe. H. D., and Wenig, M. (1945). Amer. J. Orthodont

(Oral Surg. Sect.) 31, 265.
Weinmann, J. P., and Sicher, H. (1947). Bone and Bones. Kimpton $\mathrm{N}$ London. 